University of Nebraska - Lincoln

DigitalCommons@University of Nebraska - Lincoln

July 2006

\title{
The interface electronic structure of thiol terminated molecules on cobalt and gold surfaces
}

\author{
A. N. Caruso \\ University of Nebraska-Lincoln, carusoan@umkc.edu \\ Ligen Wang \\ University of Nebraska-Lincoln, Iwang6@unl.edu \\ Sitaram Jaswal \\ University of Nebraska, sjaswal1@unl.edu \\ Evgeny Y. Tsymbal \\ University of Nebraska-Lincoln, tsymbal@unl.edu \\ Peter A. Dowben \\ University of Nebraska-Lincoln, pdowben@unl.edu
}

Follow this and additional works at: https://digitalcommons.unl.edu/physicsdowben

Part of the Physics Commons

Caruso, A. N.; Wang, Ligen; Jaswal, Sitaram; Tsymbal, Evgeny Y.; and Dowben, Peter A., "The interface electronic structure of thiol terminated molecules on cobalt and gold surfaces" (2006). Peter Dowben Publications. 81.

https://digitalcommons.unl.edu/physicsdowben/81

This Article is brought to you for free and open access by the Research Papers in Physics and Astronomy at DigitalCommons@University of Nebraska - Lincoln. It has been accepted for inclusion in Peter Dowben Publications by an authorized administrator of DigitalCommons@University of Nebraska - Lincoln. 
Published in Journal of Materials Science. Published online July 6, 2006.

Copyright (C) 2006 Springer Science + Business Media, LLC. Used by permission. DOI 10.1007/s10853-006-0362-7

\title{
InTERface Science
}

\section{The interface electronic structure of thiol terminated molecules on cobalt and gold surfaces}

\author{
A. N. Caruso, L. G. Wang, S. S. Jaswal, E. Y. Tsymbal, and P. A. Dowben \\ Department of Physics and Astronomy, Nebraska Center for Materials and Nanoscience, Behlen Laboratory of Physics, \\ University of Nebraska-Lincoln, 880111, Lincoln, NE 68588-0111, USA. Email: pdowben@unl.edu
}

\begin{abstract}
The bonding strength and interfacial electronic properties of biphenyldimethyldithiol (HS$\left.\mathrm{CH}_{2}-\mathrm{C}_{6} \mathrm{H}_{4}-\mathrm{C}_{6} \mathrm{H}_{4}-\mathrm{CH}_{2}-\mathrm{SH}\right)$ adsorbed on $\mathrm{Au}(111)$ and polycrystalline cobalt are identified from combined photoemission and inverse photoemission. In order to develop a better understanding of the thiol functional group to metal surface interaction, the stable orientation, bonding site, bonding strength and interfacial electronic properties of methylthiol $\left(\mathrm{S}-\mathrm{CH}_{3}\right)$ adsorbed on $\mathrm{Au}(111)$ and $\mathrm{Co}(0001)$ have been determined by ab initio density functional calculations. Both experiment and theory suggest that thiol bonding to cobalt surfaces is stronger compared to gold surfaces. The transfer of charge toward the adsorbed sulfur is greater for the thiols on cobalt than on gold.
\end{abstract}

\section{Introduction}

Organic "self assembled" molecular monolayers have been considered as useful electronic materials for decades [1-6], while the idea of using organic materials as dielectric layers has undergone more than a century of demonstrated application and practice. The ability to utilize the unique structural and electronic properties of self-assembled monolayers (SAMs) in nano scale electronics is more recent and has rarely included consideration of spin. Nonetheless, new dielectric barrier layers for magneto-resistive junctions are being aggressively sought [7] and organic layers may provide many unique opportunities [815] in spite of their many difficulties [16-18].
While commonly used in ferromagnet/insulator/ferromagnet magneto-resistive junctions [19, 20], oxide dielectrics are plagued with defects [21-23] and strong interface reactions that often lead to physical (i.e. structural), chemical and electronic complexities at the interface with the ferromagnetic layers [21, 24-27]. Novel non-oxide, inorganic insulating materials such as $\mathrm{BN}$ [28], ZnSe [29-33], AlAs [34, 35], AlN [36, 37], $\mathrm{C}_{2} \mathrm{~B}_{10}$ [38] and EuS [39] have been considered as potential dielectric barrier layers in magneto-resistive junctions. Unfortunately, as is the case with metal-oxides, these inorganic "non-oxide" dielectric materials are plagued by problems which include not only defects and/or structural instabilities but also temperature dependent oscillatory coupling [21,30-32] between the two ferromagnetic layers through the nominally dielectric barrier layer in the tunnel junction geometry. Only boron nitride has been experimentally shown to have chemically abrupt interfaces in which the magnetic surface states of the ferromagnet are "preserved" at the interface [4045]. It is the interface region that dominates the tunneling process [24-27, 46] and controls the spin-polarization of the tunneling current [24-27, 46, 47]. Therefore, better control of the interface electronic structure could improve device performance (greater spin selective tunneling).

If the "self assembled" organic dielectric molecular layers, or multilayers can be prepared without pinholes, such organic layers could offer one route in forming uniform dielectric layers in which some of the interface chemistry may be controlled so that interfaces between the ferromag- 
netic layers and the dielectric layers might be chemically and compositionally abrupt after fabrication. This work is part of the consideration of "soft" organic layers as a possible alternative dielectric barrier layer where the magnetic interface states might be enhanced, if not preserved. Unfortunately, this issue has not been explored extensively.

The majority of molecules being considered for the classical molecular electronic devices contain various alkane $[2,16,48]$ and polyphenyl combinations $[2,49,50]$ functionalized with endgroups such as thiols ( $\mathrm{H}-\mathrm{S}-)$, amines $\left(\mathrm{H}_{2}-\mathrm{N}-\right)$ and/or cyano $(\mathrm{C}-\mathrm{N})[2,51-53]$. Not surprisingly, the majority of experimental effort and complementary theoretical work has addressed the bonding and adsorption of these organic layers on gold surfaces, and to a lesser extent, silver surfaces. There has been little effort exploring bonding of potential organic dielectric layers to ferromagnetic surfaces [54, 55], with a few exceptions [8-15].

Oligophenyl functionalized molecules (usually with thiol or cyano end groups) provide, at present, some of the best candidates for molecular systems with a dense and regular packing of benzene rings and desired electronic properties [50]. Although very popular as candidates for junction devices, there are clearly problems associated with the use of alkane thiols $[16,55,56]$. Biphenyl and terphenyl functionalized by one or two thiols and one or two methyl groups, have been extensively investigated [55-68] by X-ray photoemission spectroscopy (XPS), reflection absorption infrared spectroscopy (RAIR), nearedge X-ray absorption fine structure spectroscopy (NEXAFS), scanning tunneling microscopy (STM), ellipsometry and advancing water contact angle.

On the basis of "fingerprinting" the reflection absorption infrared measurements of adsorbed biphenyldithiols $[49,56,65]$, it has been suggested that many of these biphenyldithiols are highly oriented with the molecular axis along the surface normal, but this supposition is not universally applicable. Although promising, biphenyldithiol was interpreted as preferentially ordering with the molecular axis along the surface normal in one study [58], Wöll and coworkers [57] and others [56] found evidence that such molecular layers are not well ordered and are without a clear preferential bonding orientation. The molecular orientation of surface adsorbed biphenyldimethyldithiol was also found to be highly disordered [57]. These latter results for biphenyldithiol and biphenyldimethyldithiol contradict the assumptions undertaken in modeling conductance measurements that include placing the molecular axis normal $[49,56,58,69-71]$ or parallel [72] with the gold surface. Nonetheless, a variety of phenomena, such as molecular "switching" [72] and quantized conductance [70], have been attributed to these molecules when used as barrier layers.

\section{Methodology}

\section{Experiment}

While photoemission does not directly reproduce the density of states, as one cannot easily take into account the consequences of matrix element effects, angle resolve photoemission combined with incident light polarization does probe the wave vector and selection rule dependence of the occupied states. The reported spectra were taken using p-polarized light (incident angle $\sim 70^{\circ}$ off normal) with a $32 \mathrm{eV}$ photon energy, from the synchrotron dispersed by a 3-meter toroidal grating monochromator at the Center for Advanced Microstructures and Devices (CAMD) in Baton Rouge, Louisiana. The electronic structure of the unoccupied states was investigated using inverse photoemission spectroscopy (IPES), by the varying kinetic energy method. For both photoemission and inverse photoemission, binding energies are reported with respect to the substrate Fermi level $\left(E-E_{\mathrm{F}}\right)$, determined from spectra taken of clean gold and tantalum in intimate contact with the substrate. The photoemission photoelectron collection angle and inverse photoemission incident electron angle were both normal to the substrate surface $(\mathrm{k} \|=0$ or $\Gamma)$, as described in detail elsewhere [68].

The substrates include the $\mathrm{Au}(111)$ surface prepared by epitaxial growth on $\mathrm{Si}(111)$, while the polycrystalline Co surfaces were prepared by thermal evaporation of Co onto the $\mathrm{Au}(111)$ surfaces. XRD was used to verify the $\mathrm{Au}(111)$ texture.

Depositions of 1,1'-biphenyl-4,4'-dimethyldithiol (BPDMT) were undertaken by solution method on the gold substrates and by adsorption from the sublimed vapor on both the gold and cobalt substrates, as described elsewhere [68]. No evidence was found for photodegradation and/or thermal desorption during the course of our measurements.

\section{Theory}

We performed spin-polarized calculations using the pseudopotential plane-wave method [73], implemented within a VASP code $[74,75]$, in the framework of density-functional theory with the generalized gradient approximation (GGA) for the exchange-correlation potential [76]. To model the surfaces we used a slab with four and five atomic layers for $\mathrm{Au}(111)$ and $\mathrm{Co}(0001)$, respectively, separated by a vacuum region equivalent to 12 atomic layers. The supercell had a $\sqrt{ } 3 \times \sqrt{ } 3 \mathrm{R} 30^{\circ}$ periodicity parallel to the surface. The adsorbed molecules were placed on the top of the slab and the two bottom Co layers of the slab were fixed at the calculated bulk lattice constant. All other atoms in the substrate and in the adsorbed organic molecule were allowed to relax. The Vanderbilt ultrasoft 
pseudopotentials [77] were employed to represent the interaction of the core and the valence electrons in these atoms. The electronic wave functions were expanded in a plane-wave basis set with an energy cutoff of $350 \mathrm{eV}$. A $6 \times 6 \times 1$ mesh of $\mathrm{k}$ points was used for the surface Brillouin zone integration.

In order to find the most stable interface structure, we calculated the adsorption energy Eads per molecule, which we defined as:

$$
\mathrm{E}_{\mathrm{ads}}=\left[\mathrm{E}_{\mathrm{s}}+n \mathrm{E}_{\mathrm{m}}-\mathrm{E}_{\mathrm{m} / \mathrm{s}}\right] / n
$$

where $\mathrm{E}_{\mathrm{s}}, \mathrm{E}_{\mathrm{m}}$, and $\mathrm{E}_{\mathrm{m} / \mathrm{s}}$ are the total energies of the clean substrate, the gas phase molecule and the adsorbate molecule - substrate system respectively and $\mathrm{n}$ is the number of adsorbed molecules.

\section{Thiol-metal bonding and perturbation of the molecu- lar orbitals}

The experimental molecular orbital binding energies provide an indication that biphenyldimethyldithiol is bound more strongly to cobalt than to gold [68]. For the thicker films, deposited from solution, the gold substrate photoemission and inverse photoemission features are completely suppressed and the photoemission spectra are dominated by photoemission features whose origin are the BPDMT molecular orbitals (Figs. 1a and 2). With submonolayer coverages of BPDMT (formed by adsorption from the vapor), the substrate gold photoemission features are only weakly suppressed (Fig. 1b). For BPMDT deposited on cobalt (formed by adsorption from the vapor), there is more suppression of the substrate cobalt features, with only the Co $3 \mathrm{~d}$ bands evident near the Fermi level (Fig. 1c).

From previous work [68], we can identify a number of molecular orbitals of biphenyldimethyldithiol (BPDMT). The expected molecular orbitals of 1,1'-biphenyl-4,4'-dimethyldithiol thin films have been observed in the combined photoemission/inverse photoemission spectra. The chemical potential adjusts to place the Fermi level closer to the lowest molecular orbital (LUMO) than the highest occupied molecular orbital (HOMO) providing a HOMOLUMO gap of $7.8 \mathrm{eV}$ [68], as seen in Fig. 2. Using these prior assignments, the most obvious features in the photoemission spectra due to adsorbed 1,1'-biphenyl-4,4'-dimethyldithiol alone are traced by the three dotted lines, representing the HOMO 2, HOMO 4+5 and HOMO 6+7 molecular orbitals respectively, as indicated in Fig. 1.

By comparing spectra for submonolayer molecularly adsorbed species on different substrates, differences in the common molecular orbital binding energies can be observed. In some cases, the molecular orbitals of very thin films are better identified in difference spectra (spectra taken after adsorption, with the photoemission spectra of the clean metal substrate subtracted). In Fig. 1, the difference spectra 1d (gold) and 1e (cobalt), exhibit BPDMT molecular orbitals binding energies that strongly depend upon substrate. For photoemission features, derived from the many molecular orbitals of BPDMT, there is a $\sim 1.7 \mathrm{eV}$ binding energy shift toward greater binding energy for submonolayer coverages on the cobalt surface as compared to gold. This binding energy shift of the molecular orbitals for cobalt surfaces (Fig. 1c and e) is evident when compared to both ultra thin coverages of BPDMT on gold (Fig. 1b and d) and very thick coverages of BPDMT on gold formed by deposition from solution (Fig. 1a). While this apparent shift of the molecular orbitals for BPDMT on cobalt towards greater binding energies is more pronounced when comparing with thicker BPDMT films on gold, we can safely assume that this molecular orbital

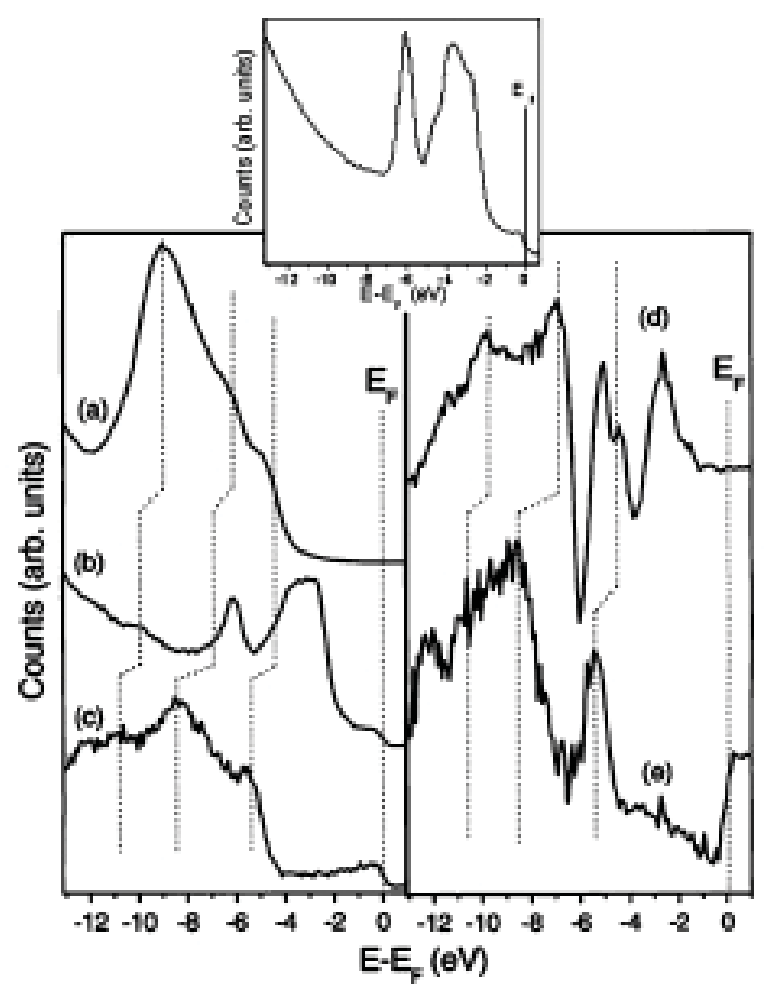

Fig. 1 Photoemission spectra of the occupied states of biphenyldimethyldithiol adsorbed on gold by solution (a) by vapor (b) and on cobalt by vapor (c) as a function of binding energy referenced to the Fermi level (LEFT). Difference spectra between the clean substrate and vaporadsorbed biphenyldimethyldithiol, normalized to background, on gold (d) and on cobalt (e) are shown (RIGHT) to highlight the biphenyldimethyldithiol molecular orbital contributions. In both panels, the dotted lines trace out the common molecular orbitals which show a shift toward higher binding energy for adsorptions on cobalt relative to gold. There is also a shift toward higher binding energy for BPMDT adsorbed on gold by vapor (a) over solution (b). The photoemission spectra of clean gold is shown in the inset. 


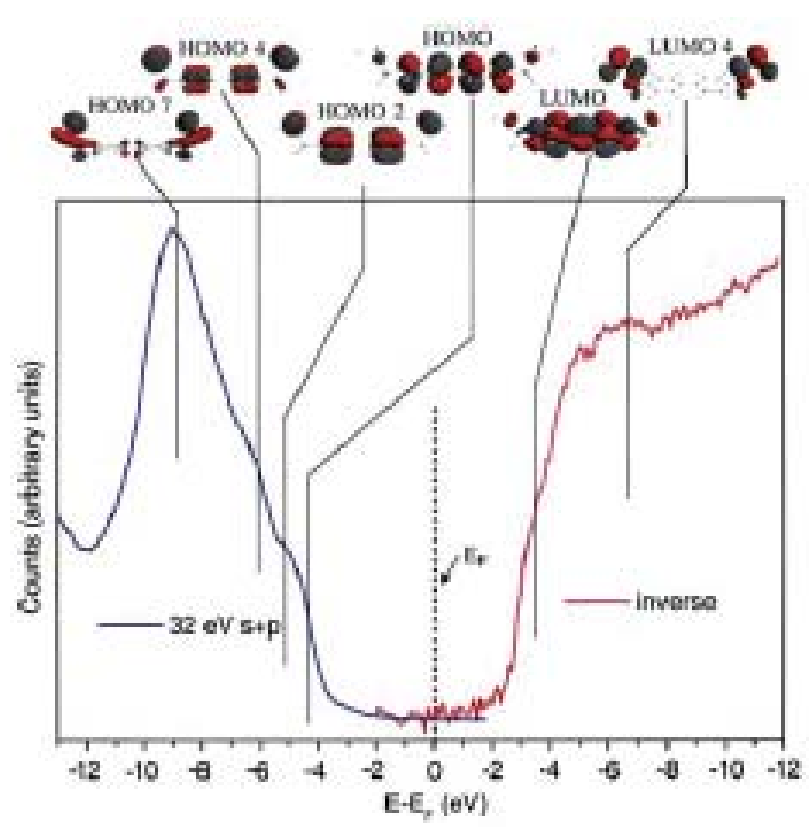

Fig. 2 Occupied (blue) and unoccupied (red) molecular orbital contributions of 1,1'-biphenyl-4,4'-dimethanethiol deposited on Au from solution to the photoemission and inverse photoemission respectively. The molecular orbitals are assigned adopting the $\mathrm{C} 2 \mathrm{~V}$ symmetry of the isolated molecule, with those forbidden by photoemission (a2 symmetry) not shown. Deposition from solution led to formation of molecular films about 5 or more monolayers thick, that were seen to be well ordered on $\mathrm{Au}(111)$, as discussed in [68].

binding energy shift will be evident when comparing the cobalt spectra with all intermediate coverages of BPDMT on gold.

This is related to the bonding effects at the organicmetal interface, i.e. this is an initial state chemical effect associated with BPDMT bonding with the cobalt surface. The hybridization is stronger at the Co surface than at the $\mathrm{Au}$ surface resulting in a charge redistribution at the interface producing a larger electric polarization at the Co surface than at the Au surface. This difference in electrostatic potential leads to the experimentally observed $\sim 1.7 \mathrm{eV}$ binding energy shift toward greater binding energy for S and C ' $\mathrm{s}$ ' and ' $\mathrm{p}$ ' states on Co compared to $\mathrm{Au}$ (Fig. 1), and explained theoretically below. Contributions from photo-induced charging, decomposition or symmetry breaking due to different substrate surfaces can be excluded on the basis of photoemission and inverse photoemission $[68,78]$. Although final state photoemission effects can nonetheless exist, for these very thin overlayers of BPDMT, we can safely assume that the metal substrate will screen the photoemission final state sufficiently so that such final state effects could not account for more than a very small fraction of the observed substrate dependent binding energy shifts.

In general, the larger occupied bonding to unoccupied antibonding orbital gap, the greater the bond strength
[54]. Therefore, larger bonding orbital binding energies, attributable to initial state chemical effects indicate a stronger bonding configuration. The shift to higher binding energies that accompanies stronger adsorbate - metal bonds need not affect all molecular orbitals equally, as is the case here. The classic example of dinitrogen (N2) adsorption on metal surfaces is a more extreme example of some molecular orbitals (notably the nitrogen $2 \sigma$ and $3 \sigma$ molecular orbitals) shifting to greater binding energies $[79,80]$, associated with stronger adsorbate interactions, but in many other N2 adsorption on metal surfaces respects qualitatively similar to the results obtained for BPDMT [54]. The increased molecular binding energies for BPDMT films on cobalt are clear evidence that this biphenyl-dithiol forms a stronger bond on cobalt than on gold. This surmise is strongly supported in the theory, as discussed below.

\section{Substrate dependent bonding sites}

Because the region of key importance is the interface between the thiol molecule and metal substrate, a smaller thiol functionalized molecule, methylthiolate, has been chosen as a starting model for investigation of the metal/ thiol interface electronic structure. First-principles calculations of methylthiol were performed for adsorption on $\mathrm{Au}(111)$ and $\mathrm{Co}(0001)$ surfaces.

The calculations initially determined, through adsorption energy comparisons, the most stable bonding site. The atop, bridge and hollow fcc and hep sites were considered for methylthiolate adsorption on both the gold and cobalt surfaces. The most stable site for methylthiol adsorption on $\mathrm{Au}(111)$ is close to the bridge site (as found by others $[81,82]$ ), but the hollow fcc and hep sites provide the minimum energy on cobalt [83]. For these stable sites on both metals, the $\sqrt{ }^{-} 3 \times \sqrt{-} 3$ adlayer ordered structure was calculated as the most favorable. The optimized geometry (orientation) and the most stable bonding site of methylthiol is shown in Fig. 3. Orientation of the sulfur to carbon bond of the methylthiol on $\mathrm{Co}(0001)$ in the hcp hollow site is normal to the surface but in the case of adsorption on $\mathrm{Au}(111)$ in the bridge site, the sulfur to carbon bond angle is canted at 54.1 from surface normal.

With this foundation as to the simple thiol adlayer site and orientation, we calculated the local density of states (LDOS) for methylthiol, adsorbed on each metal and as a free layer, summarized in Fig. 4. As is evident from Fig. $4 \mathrm{~b}$ and $\mathrm{c}$, there is a significant binding energy difference between common molecular orbitals of methylthiol for adsorption on gold relative to cobalt of approximately $0.9 \mathrm{eV}$ to $1.3 \mathrm{eV}$. These substrate dependent binding energy shifts are very similar to those observed in experiment for the large and more complex biphenyldimethyldithiol (Fig. 1). Although qualitative, we can say that the photo- 


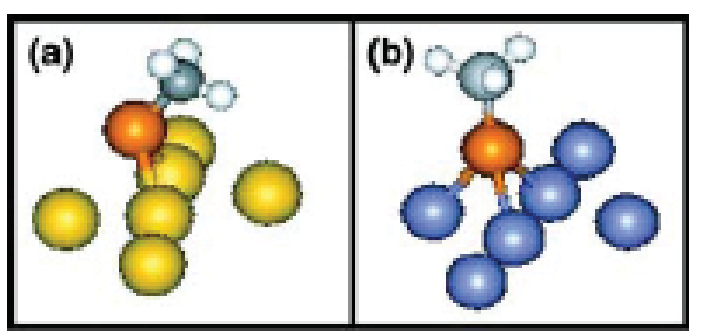

Fig. 3 A schematic illustration of the calculated minimum energy bonding site and bond angle between the sulfur and carbon of methylthiol on $\mathrm{Au}(111)$ (a) and on $\mathrm{Co}(0001)(\mathrm{b})$

emission features due to the HOMO 2, HOMO 4+5 and HOMO $6+7$ of biphenyldimethyldithiol [68] exhibit substrate dependent shifts roughly similar to the relative ground state binding energy positions of the localized molecular orbitals with strong sulfur S-3p, carbon C-2s and sulfur S-3s spectral weight of methylthiol, as labeled in Fig. 4.

A key result of our calculations is that the adsorption energy of the $\mathrm{CH}_{3} \mathrm{~S}$ thiolate on $\mathrm{Co}(0001)$, about $3 \mathrm{eV}$, is much higher than the adsorption energy of the $\mathrm{CH}_{3} \mathrm{~S}$ thiolate on $\mathrm{Au}(111)$, about $0.5 \mathrm{eV}$. This mirrors our conclusions about the hybridization that we inferred from the binding energy shifts for biphenyldimethyldithiol adsorption, discussed above.

Another similarity between experimental results obtained for biphenyldimethyldithiol and the theoretical results obtained for methythiolate on each substrate is the perturbation to the surface layer density of $d$ orbital states due to adsorption. From experiment, the gold 5d orbitals

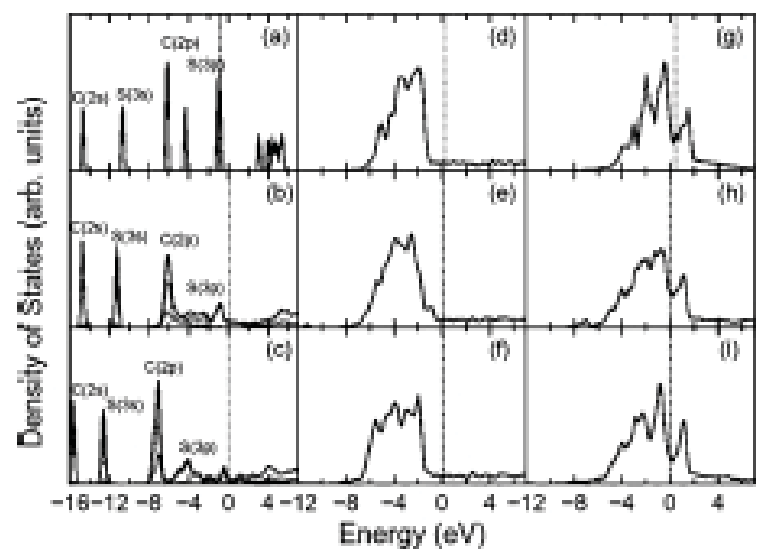

Fig. 4 Density of states for methylthiol (a) as a free layer; (b) adsorbed on $\mathrm{Au}(111)$; (c) adsorbed on $\mathrm{Co}(0001)$. Density of states for $\mathrm{Au}(111)$ (d) clean 1st layer; (e) 1st layer with adsorbate; (f) 2nd layer with adsorbate. Density of states for $\mathrm{Co}(0001)(\mathrm{g})$ clean 1st layer; (h) 1st layer with adsorbate; (i) 2 nd layer with adsorbate. The dotted line denotes the Fermi level placement at zero binding energy. The partial local density of states contributions from sulfur $3 p$ (blue), carbon $2 s$ (red), sulfur $3 \mathrm{~s}$ (green), carbon $2 \mathrm{p}$ (dashed), and total (filled dark line) are indicated. at 3- and 6-eV binding energy and the cobalt $3 \mathrm{~d}$ orbitals near the Fermi level are suppressed even with submonolayer adsorption of BPDMT. The same density of states reduction is observed from calculation as shown between the clean and methylthiol adsorbed first (frontier) layers of $\mathrm{Au}(111)$ (Fig. 4d, e) and $\mathrm{Co}(0001)$ (Fig. 4g, h). This density of states perturbation to the gold and cobalt $\mathrm{d}$ orbitals is also reflected by the charge density differences (Fig. 5).

The binding site, found from our calculations for methylthiol on $\mathrm{Au}(111)$, is well off the bridge site which is not actually one of the sites suggested by recent X-ray standing wave [84] or photoelectron diffraction [85] experiments. Deviations from the atop site are not completely precluded by that data. If the binding site for methylthiol on $\mathrm{Au}(111)$ is actually the atop site, as suggested by these experiments $[84,85]$, then the comparison between methylthiol on gold and cobalt should be even more dramatic as discussed below. Our calculated sulfur to carbon bond angle, of $54.1^{\circ}$, from surface normal, is in agreement, however, with the experimental value of $50^{\circ} \mathrm{ob}-$ tained from photoelectron diffraction experiments [85].

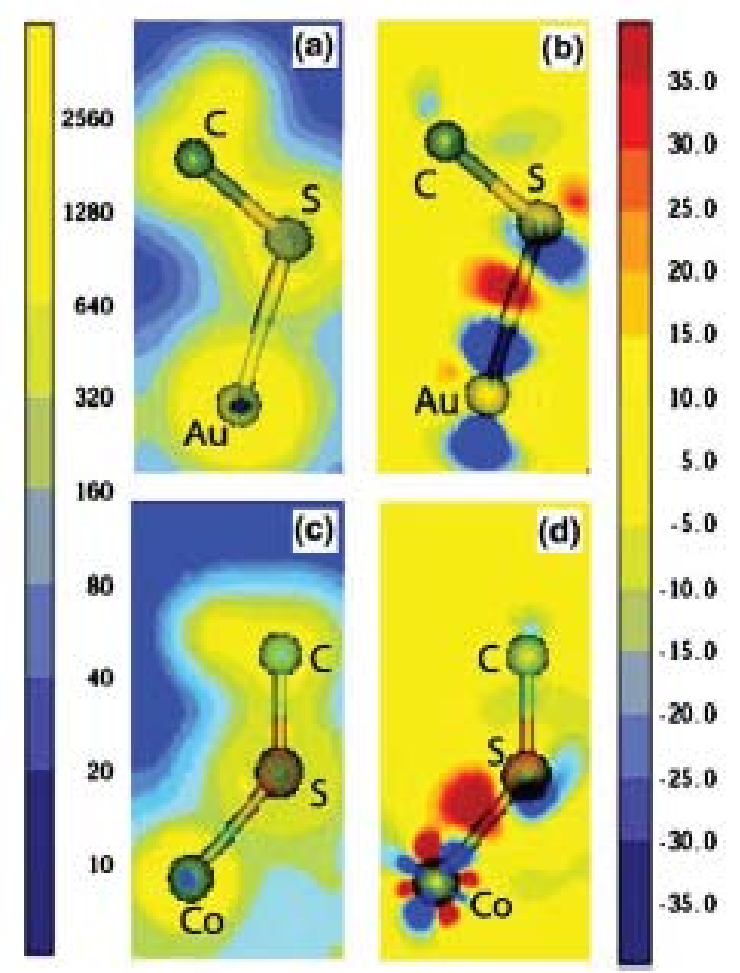

Fig. 5 Total valence electron density for methylthiol adsorbed on gold (a) and cobalt (c) and difference electron density on gold (b) and cobalt (d). Both densities are calculated for the optimized bonding site and sulfur to carbon orientation. The difference densities (b,d) is $\Delta \rho=\rho\left(\mathrm{CH}_{3} \mathrm{~S} /\right.$ substrate $)-\rho$ (substrate) $-\rho\left(\mathrm{CH}_{3} \mathrm{~S}\right)$ for which the $\rho\left(\mathrm{CH}_{3} \mathrm{~S}\right)$ is from the gas phase whereas the $\rho\left(\mathrm{CH}_{3} \mathrm{~S} /\right.$ substrate) is the interfacial state (perturbation) formation. 
While the photoemission binding energy shifts between BPDMT adsorbed on gold and cobalt are reflected in the calculations for methylthiol adsorption, the orientation of BPDMT may perturb both the binding site and the sulfur carbon bond orientation. Few molecular orbitals can be observed for BPDMT adsorbed on gold from the vapor, without taking a difference spectra (subtracting the gold photoemission features as was done in Fig. 1d). This makes the preferential molecular orientation more difficult to identify from the light polarization effects in photoemission [68]. Nonetheless, enhancement was evident with p-polarized light for the photoemission feature resulting from the HOMO-6 and HOMO-7 molecular orbitals, as described elsewhere. Enhancement in spolarized light is observed for the HOMO-4 and HOMO5 photoemission feature. Such light polarization effects, although small and difficult to identify, are more consistent with the long molecular BPDMT axis and benzene ring planes parallel with the surface [68]. Unfortunately, because only a limited number of BPDMT photoemission features are easily identified, we cannot completely exclude the possibility that the molecule is adsorbed with the benzene ring plane(s) perpendicular to the surface and the long molecular axis parallel with the surface, though the latter configuration seems unlikely. Because there is very little dependence of the photoemission spectra on light polarization for BPDMT adsorbed from the vapor on cobalt, little can be said about preferential orientation of the molecule on this surface [68].

As noted in the introduction, attempts have been made to use RAIRS to assign the molecular orientation for dithiol species similar to BPDMT [57-59]. Assignments were made by analysis of benzene ring vibrational modes intensities and position. In one study, the molecular orientation of the biphenyldithiol was interpreted as upright (the molecular axis along the surface normal) [59], and disordered in the other two $[57,58]$. As a result of data showing band structure effects in the dispersion of the molecular orbitals [68], prior studies of BPDMT multilayers [68] find strong evidence of an ordered packing of the molecular film, with the long molecular axis parallel with the surface.

\section{Charge redistribution at the interface}

Thiol terminated molecular adsorption on metal surfaces is complicated by the substrate dependent bonding situation, which is neither purely ionic nor purely covalent as is evident from the charge distributions in the interface region, as shown in Fig. 5. The key to understanding this type of bonding is from the study of the interface density of states in light of the charge redistribution due to the extent and manner of the orbital hybridization with the substrate.

The charge density differences, shown in Fig. 5b,d, explicitly illustrate hybridization between the methylthi$\mathrm{ol}$ and the gold and cobalt substrates. The electron charge transfer calculated within an atomic sphere of $1.16 \AA$ around the sulfur atom suggests that there is a $0.22 \mathrm{e}^{-}$donation from cobalt compared to a $.09 \mathrm{e}^{-}$donation from gold to sulfur. This implies more extensive hybridization in the case of the Co substrate compared to the Au substrate. The greater charge transfer result is an increase of the dipole layer at the interface which leads to an electrostatic potential step causing the position of the molecular orbital energy levels of the thiol molecule to shift with respect to the Fermi energy. Due to the larger charge transfer and concomitant stronger electric polarization at the thiol/cobalt interface, the change in electrostatic potential is more significant resulting in higher binding energies of the thiol molecular orbitals on cobalt than is the case on gold (Fig. 4b, c). Large bond length differences also exist: we calculated a Co-S thiol bond length of $2.22 \AA$ that is far smaller than the calculated Au-S $2.52 \AA$ bond length. The calculated shorter cobalt to sulfur bond length and greater calculated charge donation relative to the $\mathrm{Au}-$ $\mathrm{S}$ thiol adsorbate bond are further indications that thiols bond more strongly to cobalt than gold substrates. The charge donation results are consistent with basic electronegativity arguments or the generally greater reactivity of cobalt surfaces.

Although indirect, it was found [68] that the BPDMT lowest unoccupied molecular orbital is placed, in inverse photoemission, approximately $1.0 \mathrm{eV}$ closer to the substrate Fermi level than the highest occupied molecular orbital (as indicated in Fig. 2); this provides some secondary evidence that the larger thiol terminated molecules are accepting (abstracting) charge from the substrate even in multilayer molecular films [37]. The resulting biphenyldimethyldithiol dielectric layer resembles a donor (ntype) like insulator.

It follows from our calculations that the 2nd (subsurface) layer of each metal substrate is nearly unaffected by thiol adsorption, rendering a close to bulk like character just below the surface of the substrate. It is typical for metals that a charge density perturbation is screened at a distance of the order of the lattice constant due to a high electron concentration in most metals. This makes the perturbation, resulting from the adsorption of a thiol, largely localized to the interface. For the $\mathrm{Co}(0001)$ substrate, our calculations predict that the surface Co atoms have a magnetic moment of $1.67 \mu_{\mathrm{B}}$, which is close to the calculated bulk value of $1.61 \mu_{\mathrm{B}}$. The $\mathrm{Co}-\mathrm{S}$ bonding and the exchange splitting of the Co $3 \mathrm{~d}$ bands induces a small magnetic moment of $0.08 \mu_{\mathrm{B}}$ at the sulfur atom. We find, 
therefore, that the $\mathrm{Co}-\mathrm{S}$ bonding at the $\mathrm{Co} /$ organic layer interface does not quench the interface magnetism. This is similar to the predictions obtained for the ideal $\mathrm{Co} /$ $\mathrm{Al}_{2} \mathrm{O}_{3}$ interface in magnetic tunnel junctions [86]. The moment distribution at the $\mathrm{Co} /$ thiol interface and altered potential across the interface will have an influence in any spin electronic application [87, 88]. Significant enhancements of polarization with cobalt sulfur bonds have been predicted [89, 90].

\section{Site symmetry and bonding orbital hybridization}

The differences in the molecular orbital hybridization with the cobalt and gold surfaces affect the manner of the bonding. This is not simply limited to our conclusions that thiol terminated molecules bond more strongly to cobalt than to gold surfaces. There are two major contributions to the differences in molecular orbital hybridization with the substrate: local site symmetry and binding energy overlap with the substrate frontier orbitals.

There is a binding energy match of the sulfur $3 p$ states at $-4 \mathrm{eV}$ binding energy in the free layer of methylthiol (Fig. 4a) with the clean gold 5d (Fig. 3d). For cobalt, the sulfur $3 p$ states closer to the Fermi level overlap with the cobalt $3 \mathrm{~d}$ (Fig. $4 \mathrm{~g}$ ). In addition, there is some binding energy overlap between the sulfur $3 p$ states at $-4 \mathrm{eV}$ binding energy and cobalt $3 \mathrm{~d}, 4 \mathrm{~s}$ and $4 \mathrm{p}$ weight states. In the case of cobalt, inspection of the cobalt band structure suggests that the energy overlap is greatest with bands of cobalt $\mathrm{p}_{x, y}, \mathrm{~d}_{x z, x y}$ character. As noted above, theory [83] suggest that the thiol binding site on $\mathrm{Co}(0001)$ is in the three fold hollow site preserving the local $\mathrm{C}_{3 \mathrm{v}}$ point group symmetry of the surface.

With this point group symmetry and binding site, the orbital hybridization sulfur $3 \mathrm{p}_{x, y}$ states with the cobalt $\mathrm{d}_{x z, x y}$ band states are favored. This results in a concomitant shift in spectra weight density from states of $\mathrm{a}_{1}$ symmetry character $\left(\mathrm{s}, \mathrm{p}_{z}, \mathrm{~d}_{3 z^{2}-r^{2}}\right.$ to e symmetry character $\left(\mathrm{p}_{x, y}\right.$, $\left.\mathrm{d}_{x^{2}-y 2}, \mathrm{~d}_{x y}, \mathrm{~d}_{x z}, \mathrm{~d}_{y x}\right)$, using the irreducible representations of the $\mathrm{C}_{3 \mathrm{v}}$ point group. This is supported by the changes in charge density contours shown in Fig. 5 d, where $\mathrm{d}_{x z}$, $\mathrm{d}_{y x}$ character (blue) from cobalt is shown to create a bond (red) with the thiol. The symmetry match and shorter bond length to the sulfur, compared to thiol adsorption on gold means that not only is the hybridization greater but that the strength of the interaction is much larger [91].

Bonding of chalcogens (including sulfur) [92] and the larger halogens [93] on fcc $3 \mathrm{~d}$ transition metals can also exhibit bonding with the metal substrate through the $\mathrm{p}_{x, y}$ orbitals rather than $\mathrm{p}_{z}$. Binding site is partly responsible: orbital overlap in the high symmetry site between main group $\mathrm{p}_{z}$ and substrate $\mathrm{d}_{3 z^{2}-r^{2}}$ is weak as the adsorbate is large and the spatial extent of $d_{3 z^{2}-r^{2}}$ from the subsurface layer is insufficient for significant overlap with the adsorbate orbitals. If adsorbate $\mathrm{s}$ or $\mathrm{p}_{z}$ character orbitals hybridization with substrate $\mathrm{s}, \mathrm{p}_{z}$ and $\mathrm{d}_{3 z^{2}-r^{2}}$ states were to dominate bonding, then the bonding would favor the high symmetry low coordination atop site, as occurs with the noble gas adsorbates [94], and has been suggested for methylthiol adsorption in $\mathrm{Au}(111)$ [84, 85].

In the case of thiol adsorption on $\mathrm{Au}(111)$, theory suggests that a very low symmetry local bonding site, well off the ideal bridge site position is favored. In this low symmetry-bonding site, there are few symmetry restrictions to bonding and though $\mathrm{d}_{z^{2}}$ like character (blue) orbitals from gold may dominate the bonding (Fig. 5), states of all rectangular representations may contribute to the bonding. The competition is between maximizing the coordination of the bonding site, and the need to adopt a low symmetry-bonding site to maximize the gold frontier orbital contributions in bonding to the thiol. With the adsorption site as the atop site of $\mathrm{Au}(111)$, as suggested by experiment $[47,48], \mathrm{d}_{z^{2}}$ like character (blue) orbitals from gold will certainly dominate the thiol bonding to the substrate, though the large cant angle of the S-C bond (about $50^{\circ}$ off normal as suggested by experiment [85] and the theory effort reported here) will continue to lower to local point group symmetry from $\mathrm{C}_{6 \mathrm{v}}$ to a very low local symmetry.

The competition between symmetry preservation and orbital character contributions to the bonding, not to mention the degree of coordination [84, 94], affect bonding site, strength of the bond and subsequent orientation of the alkane, or phenyl groups attached to the sulfur.

\section{Conclusion}

We have shown that the transition metal cobalt offers dramatically different bonding sites, orientations, bonding strengths, interfacial hybridization and charge transfer compared to adsorbing thiol terminated molecules on gold. The thiol terminated molecules biphenyldimethyldithiol and methylthiol have been studied respectively by experiment and theory for adsorption on both cobalt and gold, observing a stronger bonding to cobalt over gold. The newly formed states due to adsorption of methylthi$\mathrm{ol}$ are very similar for gold and cobalt in terms of $\mathrm{s}-\mathrm{d}$ and $\mathrm{p}-\mathrm{d}$ hybridization, yet the charge transfer amounts and resulting interfacial electronic structure are very different for the two metal substrates, which cannot be easily explained by valence arguments alone. For both cobalt and gold, only the topmost layers are noticeably perturbed from the bulk with thiol adsorption. Of interest to conduction, the $\mathrm{d}$ weight contribution to the sulfur atom from 
gold or cobalt hybridization indicates that this end group is valuable for functionalizing organic barrier layers with arene ligands. There is now promise that new insulating materials for spintronic applications may be developed, exploiting spin dependent scattering at available interface states [19, 24-27, 46]. Further, these sulfur-terminated molecules provide an excellent electronic comparison with oxide based insulating materials, but are very different structurally as they are very stable, stoichiometrically, at an interface.

The highest occupied (HOMO) to lowest unoccupied (LUMO) molecular orbital gap of the thick (multilayer) 1,1'-biphenyl-4,4'-dimethyldithiol films deposited from solution is about $7.8 \mathrm{eV}$ from the vertical energies derived from the combined photoemission and inverse photoemission spectra (Fig. 2). This is much smaller than the theoretical value of $10.33 \mathrm{eV}$ [68]. There is an absolute gap of $\sim 5.5 \mathrm{eV}$ as indicated by the absence of any density of states in the combined photoemission and inverse photoemission of BPDMT deposited from solution on gold (Fig. 2). This thermal band gap is considerably less than the HOMO LUMO gap, but clearly shows that a condensed film of BPDMT is a wide band gap insulator. This bandgap is considerably larger than the measured gap (less than 2-4 eV) determined by molecular conductance spectroscopy of similar molecules [49, 69-72].

\section{Acknowledgments}

This work was supported by the National Science Foundation through grant CHE-0415421 and the NSF “QSPINS” MRSEC (DMR-0213808), as well as the W.M Keck Foundation. Calculations were completed utilizing the Research Computing Facility of the University of NebraskaLincoln. The authors would like to thank R. Rajesh and J. Redepenning for providing the 1,1'-biphenyl-4,4'-dimethyldithiol (BPDMT) source molecule used in the experiments.

\section{References}

1. Ulman A (1991) An introduction to ultrathin organic films. Academic Press, San Diego

2. Swalen D, Allara DL, Andrade JD, Chandross EA, Garoff S, Israelachvili J, McCarthy TJ, Murray R, Pease RF, Rabolt JF, Wynne KJ, Yu H (1987) Langmuir 3:932

3. Mann B, Kuhn H (1971) J Appl Phys 42:4398

4. Polymeropoulos EE, Sagiv J (1978) J Chem Phys 69:1836

5. Netzer L, Sagive J (1983) J Am Chem Soc 105:674

6. Leger A, Klein J, Belin M, Defourne D (1971) Thin Solid Films 8:R51

7. Wolf SA, Awschalom DD, Buhrman RA, Daughton JM, von Molnar S, Roukes ML, Chtchelkanova AY, Treger DM (2001) Science 294:1488

8. Nan Ce Wen, Li Ming, Feng X, Yu S (2001) Appl Phys Lett 78:2527
9. Nan Ce Wen, Li Ming, Huang JH (2001) Phys Rev B 63: 144415

10. Dediu V, Murgia M, Matacotta FC, Talini C, Barbanera S (2002) Solid State Commun 122:181

11. Xiong ZH, Wu D, Vardeny ZV, Shi J (2004) Nature 427:821

12. Xie SJ, Ahn KH, Smith DL, Bishop AR, Saxena A (2003) Physical Review B 67:125202

13. Taliani C, Dediu V, Biscarini F, Cavallini M, Murgia M, Ruani G, Nozar P (2002) Phase Transitions 75:1049

14. Fu JY, Ren JF, Liu DS, Xie SJ (2004) Acta Physica Sinica 53:1989

15. Ren JF, Fu JY, Liu DS, Xie SJ (2004) Acta Physica Sinica $53: 3814$

16. Ovchenkov YA, Geisler H, Burst JM, Thornburg SN, Ventrice CA Jr., Zhang C, Redepenning J, Losovyj Y, Rosa L, Dowben PA, Doudin B (2003) Chem Phys Lett 381:7

17. Francis TL, Mermer O, Veeraraghavan G, Wohlgenannt $M$ (2004) New J Phys 6:185

18. Salis G, Alvarado SF, Tschudy M, Brunschwiler T, Allenspach R (2004) Phys Rev B 70:085203

19. Tsymbal EY, Mryasov ON, LeClair PR (2003) J Phys Condens Mat 15:R109

20. Zutic I, Fabian J, Sarma SD (2004) Rev Modern Physics 76:323

21. Dowben PA, Doudin B (2005) In: Donath M, Nolting W (eds) Local Moment Ferromagnets: Unique Properties for Modern Applications, Lecture Notes in Physics 678, Springer-Verlag, ISBN No. 0075-8450, pp 309-326

22. Zhuravlev MYe, Tsymbal EY, Vedyayev AV (2005) Phys Rev Lett, 94: 127203

23. Cheng R, Caruso AN, Yuan L, Liou S-H, Dowben PA (2003) Appl Phys Lett 82:1443

24. Belashchenko KD, Tsymbal EY, van Schilfgaarde M, Stewart DA, Oleynik II, Jaswal SS (2004) Phys Rev B 69:174408

25. Belashchenko KD, Tsymbal EY, van Schilfgaarde M, Stewart DA, Oleynik II, Jaswal SS, (2004) J

Mater 272-276:1954

26. Oleinik II, Tsymbal EY, Pettifor DG (2001) Phys Rev B 65:020401

27. Oleinik II, Tsymbal EY (2004) Interface Sci 12:105

28. Lukaszew RA, Sheng Y, Uher C, Clarke R (1999) Appl Phys Lett 75:1941

29. Gustavsson F, George JM, Etgens VH, Eddrief M (2001) Phys Rev B 64:184422

30. Hunziker M, Landolt M (2000) Phys Rev Lett 84:4713

31. Walser P, Hunziker M, Speck T, Landolt M (1999) Phys Rev B 60:4082

32. Walser P, Hunziker M, Landolt M, (1999)J Magn Magn Mat 200:95

33. Mavropoulos Ph, Papanikolaou N, Dederichs PH (2000) Phys Rev Lett 85:1088

34. Tanaka M, Higo Y (2001) Phys Rev Lett 87:026602

35. Petukhov AG, Chantis AN, Demchenko DO (2002) Phys Rev Lett 89, art. no. 107205 
36. Yoshimura S, Shoyama T, Nozawa T, Tsunoda M, Takahashi M (2004) IEEE Trans Magnet 40:1

37. Sharma M, Nickel JH, Anthony TC, Wang SX (2000) Appl Phys Lett 77:2219

38. Bernard L, Monson J, Sokolov A, Liu Zong-Yuan, Yang C-S, Dowben PA, Doudin B, Harken A, Welsch P, Robertson BW (2003) Appl Phys Lett 83:3743

39. LeClair P, Ha JK, Swagten HJM, Kohlhepp JT, van de Vin CH, de Jonge WJM (2002) Appl Phys Lett 80:625

40. Nagashima A, Tejima N, Gamou Y, Kawai T, Oshima C (1995) Phys Rev Lett 75:3918

41. Nagashima A, Tejima N, Gamou Y, Kawai T, Oshima C (1996) Surf Sci 358:307

42. Grad GB, Blaha P, Schwarz K, Auwarter W, Greber T (2003) Phys Rev B 68, art. no. 085404

43. Oshima C, Nagashima A (1997) J Phys Cond Matter 9:1

44. Nagashima A, Tejima N, Gamou Y, Kawai T, Terai M, Wakabayashi M, Oshima C (1996) Int J Modern Phys B 10:3517

45. Greber T, Auwarter W, Hoesch M, Grad G, Blaha P, Osterwalder J (2002) Surf Rev Lett 9:1243

46. Dowben PA, Jenkin SJ (2005) In: A. Narlikar (ed) Frontiers in magnetic materials, Springer Verlag

47. Tsymbal EY, Pettifor DG (1997) J Phys Condens Mat 9:L411

48. Pflaum J, Bracco G, Schreiber F, Colorado Jr. R, Shmakora OE, Lee TR, Scoles G, Kahn A (2002) Surf Sci 498:89

49. Hong S, Reifenberger R, Tian W, Datta S, Henderson J, Kubiak CP (2000) Superlattices Microstruct 28:289

50. Robertson N, McGowan CA (2003) Chem Soc Rev 32:96

51. Reed MA, Takhee L (eds) (2003) In: Molecular nanoelectronics, American Scientific Publishers, Stevenson Ranch, California

52. Kagan CR, Ratner MA (2004) MRS Bull 29:376

53. Reed MA (2001) MRS Bull 26:113

54. Dowben PA, Jaewu C, Morikawa E, Bo Xu (2002) In: Nalwa HS (ed) Handbook of thin films, vol 2. Characterization and spectroscopy of thin films, Chapter 2, Academic Press, p 61114

55. Zharnikov M, Grunze M (2001) J Phys Condens Matt 13:11333

56. Zharnikov M, Grunze M (2002) J Vac Sci Technol B 20:1793

57. Weckenmann U, Mittler S, Naumann K, Fischer RA (2002) Langmuir 18:5479

58. Azzam W, Wehner BI, Fischer RA, Terfort A, Wöll C (2002) Langmuir 18:7766

59. Henderson JI, Feng S, Ferrence GM, Bein T, Kubiak CP (1996) Inorg Chimica Acta 242:115

60. Rong H-T, Frey S, Yang YJ, Zharnikov M, Buck M, Wühn M, Wöll Ch, Helmchen G (2001) Langmuir 17:1582

61. Henderson JI, Feng S, Bein T, Kubiak CP (2002) Langmuir 16:6183

62. Cygan MT, Dunbar TD, Arnold JJ, Bumm LA, Shedlock NF, Burgin TP, Jones L II, Allara DL, Tour JM, Weiss PS (1998) J Am Chem Soc 120:2721

63. Frey S, Stadler V, Heister K, Eck W, Zharnikov M, Grunze M (2001) Langmuir 17:2408
64. Shaporenko A, Adlkofer K, Johansson LSO, Ulman A, Grunze M, Tanaka M, Zharnikov M (2004) J Phys Chem B 108:17964

65. Tai Y, Shaporenko A, Rong H-T, Buck M, Eck W, Grunze M, Zharnokov M (2004) J Phys Chem B 108:16806

66. Shaporenko A, Brunnbauer M, Terfort A, Grunze M, Zharnokov M (2004) J Phys Chem B 108:14462

67. Shaporenko A, Adlkofer K, Johansson LSO, Tanaka M, Zharnokov M (2003) Langmuir 19:4992

68. Caruso AN, Rajesh R, Gallup G, Redepenning J, Dowben PA (2004) J Phys: Condens Matter 16:845

69. Schön JH, Meng H, Bao Z (2001) Nature 413:713

70. Schön JH, Meng H, Bao Z (2001) Science 294:2138

71. Datta S, Tian W, Hong S, Reifenberger R, Henderson JI, Kubiak CP (1997) Phys Rev Lett 79:2530

72. Rochefort A, Martel R, Avouris Ph (2002) Nanoletters 2:877

73. Payne MC, Teter MP, Allan DC, Arias TA, Joannopoulos JD (1992) Rev Mod Phys 64:1045

74. Kresse G, Hafner J (1993) Phys Rev B 47:558

75. Kresse G, Furthmüller J (1996) Comput Mater Sci 6:15

76. Perdew JP, Chevary JA, Vosko SH, Jackson KA, Pederson MR, Singh DJ, Fiolhais C (1992) Phys Rev B 46:6671

77. Vanderbilt D (1990) Phys Rev B 41:7892

78. Caruso AN, Rajesh R, Gallup G, Redepenning J, Dowben PA (2004) J Phys Chem B 108:6910

79. Shinn ND (1990) Phys Rev B 41:9771

80. Shinn ND, Tsang K-L (1990) J Vac Sci Technol A 8:2449

81. Vargas MC, Giannozzi P, Selloni A, Scoles G (2001) J Phys Chem B 105:9509

82. Hayashi T, Morikawa Y, Nozoye H (2001) J Chem Phys 114:7615

83. Wang LG, Tsymbal EY, Jaswal SS (2004) Phys Rev B 70:075410

84. Kondoh H, Iwasaki M, Shimada T, Amemiya K, Yokoyama T, Ohta T, Shimorura M, Kono S (2003) Phys Rev Lett 90:066102

85. Roper MG, Skegg MP, Fisher CJ, Lee JJ, Dhanak VR, Woodruff DP, Jones RG (2004) Chem Phys Lett 389:87

86. Oleinik II, Tsymbal EY, Pettifor DC (2000) Phys Rev B $62: 3952$

87. Mazin II (1999) Phys Rev Lett 83:1427

88. Mazin II (2001) Europhys Lett 55:404

89. Mazin II (2000) Appl Phys Lett 77:3000

90. Shishidou T, Freeman AJ, Asahi R (2001) Phys Rev B 64, art. no. 180401

91. Burdett JK (1984) Progr Sol St Chem 15:173

92. Plummer EW, Tonner B, Holzwarth N, Liebsch A (1980) Phys Rev B 21:4306

93. Dowben PA (1987) CRC Crit Rev Solid State Mater Sci 13:191

94. Da Silva JLF, Stampfl C, Scheffler M (2003) Phys Rev Lett 90, art. no. 066104 